\title{
An approximation to the errors in the planetary ephemerides of the Astronomical Almanac
}

\author{
E. M. Standish^ \\ CalTech/Jet Propulsion Laboratory, 301-150, Pasadena, CA 91109, USA \\ Received 12 November 2003 / Accepted 13 January 2004 \\ Abstract. Errors in the planetary coordinates of the Astronomical Almanac are approximated by comparing their fundamental \\ ephemerides, upon which they are based, with a more recent ephemeris. JPL's DE200 provided the fundamental planetary \\ and lunar ephemerides of the Astronomical Almanac for the years, 1984-2002, and DE405 has replaced DE200 in that role, \\ starting in 2003. Recently, new observational measurements have become available, thereby enabling further improvements in \\ the ephemerides, and thus, a measure of the previous errors.
}

Key words. ephemerides

\section{Introduction}

Planetary and lunar coordinates in the Astronomical Almanac for the years, 1984-2002, are based upon JPL's DE200; starting in 2003, DE200 was replaced by DE405 in that role. These fundamental ephemerides are improved with enhanced data sets to which they are adjusted as well as refined modeling of the dynamics and of the observations themselves.

The accuracy of an ephemeris depends upon three major factors: 1) the correctness and completeness of the equations of motion which describe the gravitational physics, 2) the integration of the equations of motion, and 3) the adjustment of the ephemerides to the existing observational data. In regard to the first two factors, the equations are believed to be correct and complete, and the integration program has been tested and shown to be sufficiently accurate.

It is the third factor which has been significantly improved since the creation of DE405 in 1997. In particular, the new measurements are highly accurate: spacecraft range and doppler points from Pathfinder on the surface of Mars in 1997, range points from MGS in orbit around Mars, 1999-2003, range points from Odyssey in orbit around Mars, 2002-2003, $\Delta$ VLBI points from both MGS and Odyssey, and CCD astrometric measurements of the outer planets and their satellites.

This paper briefly discusses the new observations and their effects upon the planetary ephemerides; discussion of the lunar ephemerides will be given in a future paper. This paper then presents comparisons of the previous ephemerides, DE200 and of DE405, with a more recent ephemeris, DE409. Assuming that a later ephemeris is a significant improvement over an earlier one, the differences between the two ephemerides provide good approximations to the errors of the earlier ephemeris. Thus, the differences, DE200-DE409 and DE405-DE409,

^ e-mail: ems@smyles.jpl.nasa.gov illustrate the errors in the Astronomical Almanac over the years, 1984-2002 and 2003 onward, respectively.

\section{Inner 4 planets: Ephemerides from ranging and $\triangle$ VLBI}

The ephemerides of the four innermost planets along with the moon and the sun are all well-known with respect to each other (inter-planet distances and angles) because of the accurate ranging observations to which the ephemerides are adjusted. Furthermore, the mean motions of the inner planets are also well-determined by fitting to just the ranging observations. For explanations of this latter fact, see Williams \& Standish (1989) or Standish \& Williams (1990).

The one feature that the ranging does not provide is the orientation of the system onto an outside reference frame. Previous to DE200, the orientation was provided by fitting to the (1950) FK4-based optical transit timings (right ascension and declination). Starting with DE200, the ephemerides were oriented onto their own inherent Earth's mean equator and dynamical equinox, as discussed by Standish (1982). Beginning with DE400, the orientation has been provided by $\triangle$ VLBI observations of spacecraft in orbit around or near to a planet. For DE405, these were the Magellan Spacecraft orbiting Venus and the Phobos Spacecraft as it approached Mars. These observations link the spacecraft to the ICRF (International Celestial Reference System), and thus the planet to the ICRF, given that the planetocentric spacecraft orbit is sufficiently well-determined with respect to the planet.

\section{Uncertainties from asteroid perturbations}

There are a few hundred asteroids which are massive enough to produce a non-negligible effect upon the motions of Mars and 
the Earth. However, the masses of the asteroids are not wellknown, so it is not possible to model their perturbations with full accuracy. As a result, away from the present epoch of accurate ranging and $\triangle \mathrm{VLBI}$, the planetary ephemerides deteriorate due to the asteroids, as discussed by Williams (1984) and by Standish \& Fienga (2002).

\section{Outer planets: Reliance on the classical optical observatons}

In contrast to those of the inner planets, the ephemerides of the outer planets rely almost entirely upon optical observations. (Jupiter is an exception, with points from Pioneers, Voyagers, Ulysses, and Galileo.) With only the optical data, the outer planet ephemerides are much less accurate than those of the inner planets. For Neptune and Pluto, a single orbital period has not yet elapsed since the improvement of the meridian circle observations introduced by the impersonal micrometer in 1911. For the outer planets, especially for the two outermost, the mean motions are quite uncertain and, consequently, so are the radial distances. Modern CCD observations pinpoint present-day directions, but the distances rely upon the older observations as well.

\section{The data fit by DE200 and DE405}

The observatonal data, to which DE200 was fit in 1979, are discussed by Standish (1990); mainly:

optical meridian transit timings (1911-77, $\sigma \sim 0$ 0. 5 ; planetary radar ranging data $(1969-78, \sigma \sim 1 \mathrm{~km})$; Mariner 9 ranges to Mars $(1971-72, \sigma \sim 25 \mathrm{~m})$; the first three years of ranges to the Viking Landers on Mars $(1976-79, \sigma \sim 10 \mathrm{~m})$.

The observations fit by DE405 in 1997 are discussed by Standish (1998):

additional optical transits, now including Pluto (1911-1996);

the complete Viking set (1976-83);

additional radar ranging (now 1969-93);

CCD observations of Uranus, Neptune, and Pluto (1995-1996);

$\triangle$ VLBI observations of the Magellan spacecraft at Venus

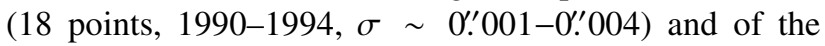
Phobos spacecraft approaching Mars (2 points, 1989, $\sigma \sim$ 0.'003, 0.'006).

\section{The most recent observations}

Since the creation of DE405 in 1997, there have been a number of additional observations to which the most recent ephemeris (DE409, March 2003) has been fit:

ranging to the Pathfinder Lander (1997; $\sigma \sim 5-10 \mathrm{~m}$; Folkner 1997);

doppler measurements to Viking and to Pathfinder (1976-1979, 1997; $\sigma \sim 0.02 \mathrm{~mm} \mathrm{~s}^{-1}$; Folkner 1997); ranging to MGS and to Odyssey (1999-2003, 2002-2003; $\sigma \sim 2-3$ m; Konopliv 2003);

$\triangle$ VLBI observations of MGS and Odyssey (2001-2003, 2002-2003; $\sigma \sim 0$.'002-0.'003, Border 2003);

CCD measurements of the outer planets and their satellites (1998-2003; 0.'05-0.'10; Stone 1998; Stone \& Harris 2000; Owen 2003).

\section{Planetary positional errors in the Almanacs, 1984-2002}

A comparison between DE200, created in 1979, and DE409, created in 2003, shows differences which must, for the most part, be attributed to the errors in DE200. As such, these differences give good estimates of the errors in the positions of the Astronomical Almanac for the years, 1984-2002, when DE200 was the basic ephemeris. Plots of the differences (in the sense, DE200-DE409) in geocentric right ascension $(\Delta \alpha)$, declination $(\Delta \delta)$, and distance $(\Delta \rho)$ are given in Figs. 1a through 9c. The four inner planets all show an offset in $\Delta \alpha$ of about -0.01 for the year 2000 with drifts of about $-0 .{ }^{\prime} 04 \mathrm{cty}^{-1}$, and in $\Delta \delta$, the differences are within \pm 0 '. 02 ; these are differences in the $10-20 \mathrm{~km}$ range. The differences in $\Delta \rho$ are less than $\pm 1 \mathrm{~km}$ over the 1970's, expanding, in general, to a few $\mathrm{km}$ by the year 2000.

The outer planets show errors which increase outward from the sun: 0. ' 1 or so during the interval of observations, but growing after that due to the mean motion errors: 0.3 cty $^{-1}$ for Jupiter on out to $2^{\prime \prime}$ cty $^{-1}$ for Neptune and $20^{\prime \prime}$ cty $^{-1}$ for Pluto.

The heliocentric coordinates of the Earth-Moon Barycenter in DE200 may be related to those in DE409, using the rotation, $\hat{\boldsymbol{r}}_{\mathrm{DE} 409} \approx \hat{\boldsymbol{r}}_{\mathrm{DE} 200}+\boldsymbol{A}(t) \times \hat{\boldsymbol{r}}_{\mathrm{DE} 200}$ where $206265 \boldsymbol{A}^{T}=\left[+0{ }^{\prime} 0022-\right.$ $\left.0{ }^{\prime} 0003 T,+0 .{ }^{\prime} 0117-0 .^{\prime} 0108 T,+0{ }^{\prime} 0123+0 .^{\prime} 0249 T\right]$ where $T$ is the number of centuries past $\mathbf{J} 2000$. The three components of the vector $\boldsymbol{A}$ represent time-dependent small rotations about the $x$-, $y$ - and $z$-axes, respectively.

Further, since the orientation of the system of the four inner planets is less well-determined than the relative angles and distances, the same rotation vector may be used for Mercury, Venus, and Mars, as well as for the Earth-Moon Barycenter. This is also evident from the similarties between the planets in the plots of Fig. 1.

\section{Planetary position uncertainties in the Almanacs starting in $\mathbf{2 0 0 3}$}

The differences, DE405-DE409, are shown in Figs. 10a-18c. Most of these are an order of magnitude smaller than those for DE200-DE409. There is a slight offset in $\Delta \alpha$ of about $0{ }^{\prime} 001$ plus a mean motion drift of about $0{ }^{\prime} 002 \mathrm{cty}^{-1}$, while $\Delta \delta$ tends to be about $\pm 0{ }^{\prime} \cdot 002$ : all differences in the $1-2 \mathrm{~km}$ range. The geocentric distances are less than 200 meters over the timespan when most of the DE405 ranges were taken.

For the outer planets, as shown in Figs. 14a-18c, agreement during the 1990's is about \pm 0 '. 05 with mean motion differences which still amount to a couple of tenths of an arcsecond per century for Jupiter, Saturn, and Uranus; substantially worse for Neptune and Pluto. 
RA : 200-409, Nerceury $=$ Earth

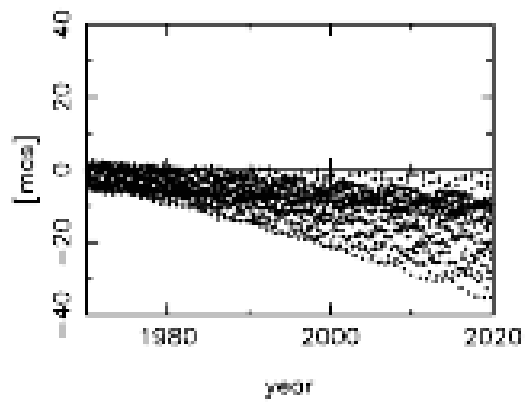

R.A. : 200-409, Venus - Earth

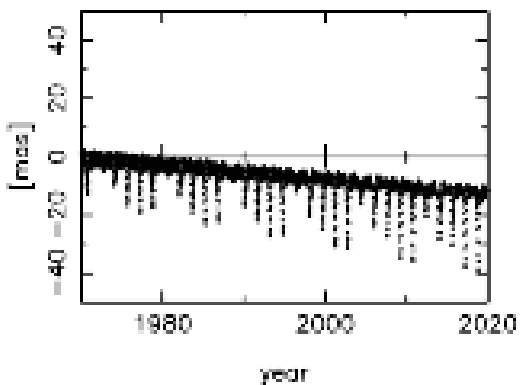

RA : 200-409, The Sun - Earth

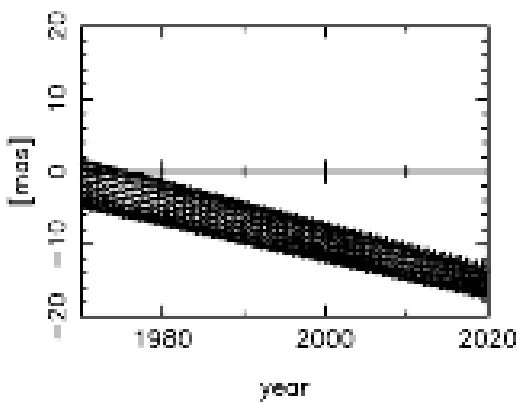

R.A. : 200-409, Wars - Earth

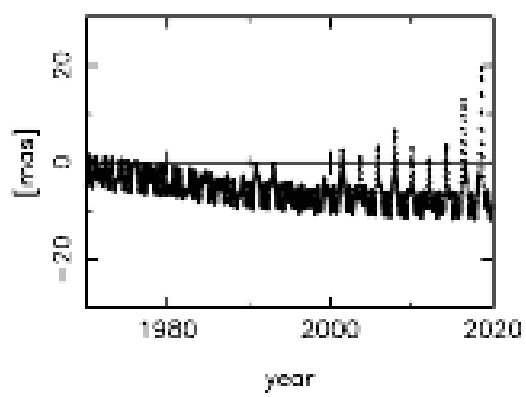

Dec, : 200-409, Nerteury $=$ Earth

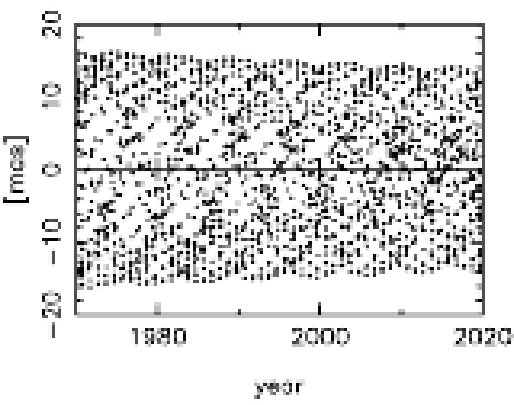

Cec. : 200-409, Venus - Eerth

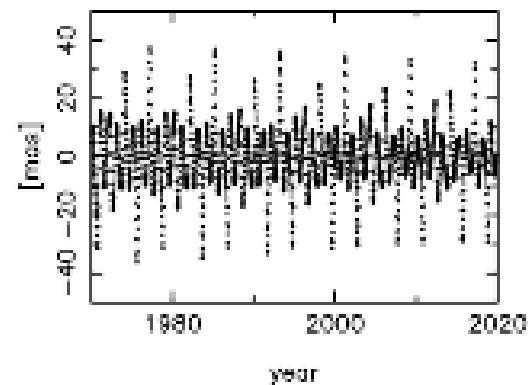

Dec. : 200-409, The Sun - Earth

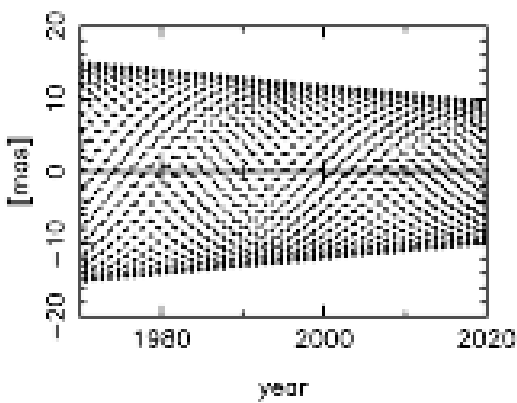

Dec. : 200-409, Mars - Earth

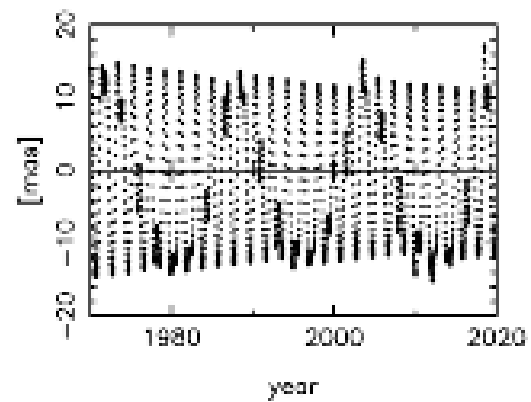

$D i s t: 200-409$, Mercen $=$ Earth

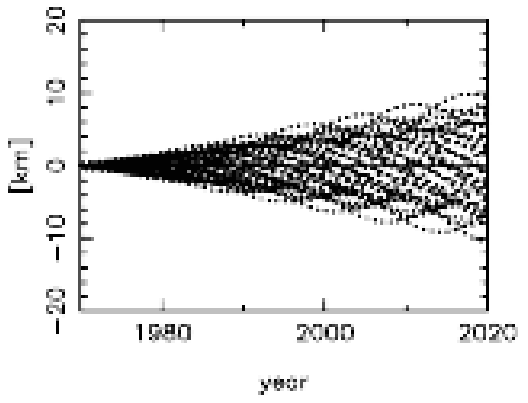

Dist : 200-409, Venus - Ecth

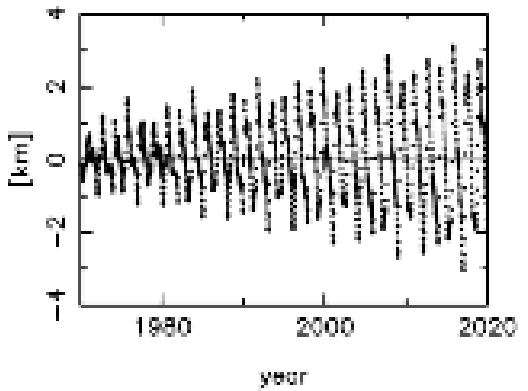

Dist : 200-409, The Sun - Earth

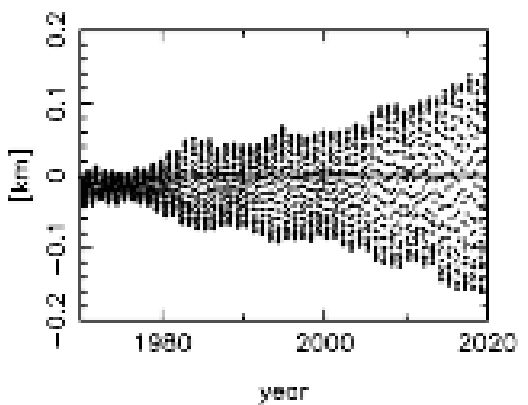

Dist : 200-409, Mars - Earth

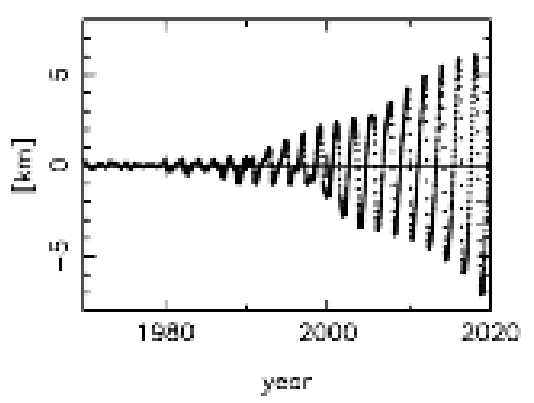

Fig. 1. The inner planet differences, DE200-DE409: effectively the errors in the Astronomical Almanac for the years, 1984-2002.

The heliocentric coordinates of the Earth-Moon Barycenter in DE405 may be related quite closely to those in DE409 as $\hat{\boldsymbol{r}}_{\mathrm{DE} 409} \approx \hat{\boldsymbol{r}}_{\mathrm{DE} 405}+\boldsymbol{A}(\mathrm{t}) \times \hat{\boldsymbol{r}}_{\mathrm{DE} 405}$ where $206265 \boldsymbol{A}^{T}=\left[+0^{\prime} \cdot 0015-\right.$ $\left.0 .{ }^{\prime} 0001 T,-0 .{ }^{\prime} 0010+0 .{ }^{\prime} 0007 T,-0.0011-0 . ' 0015 T\right]$.

\section{Discussion}

Though DE200 does not show any major problems or errors, DE405 is a definite improvement and seems to perform as well as expected. The mean motion error of DE200 $\left(\sim 00^{\prime} 04\right.$ cty $\left.^{-1}\right)$ reflects the relatively short time-span covered by the ranging data to which DE200 was fit, as well as the inclusion of perturbations from only five asteroids instead of the 300 now being used. The outer planets, relying upon optical observations only, inherited systematic errors known to exist in these data at the $0{ }^{\prime} 1$ and $1^{\prime \prime}$ cty $^{-1}$ levels. Neptune and Pluto are highly uncertain outside of the data interval, since less than a complete period has been observed for these two outermost planets. 

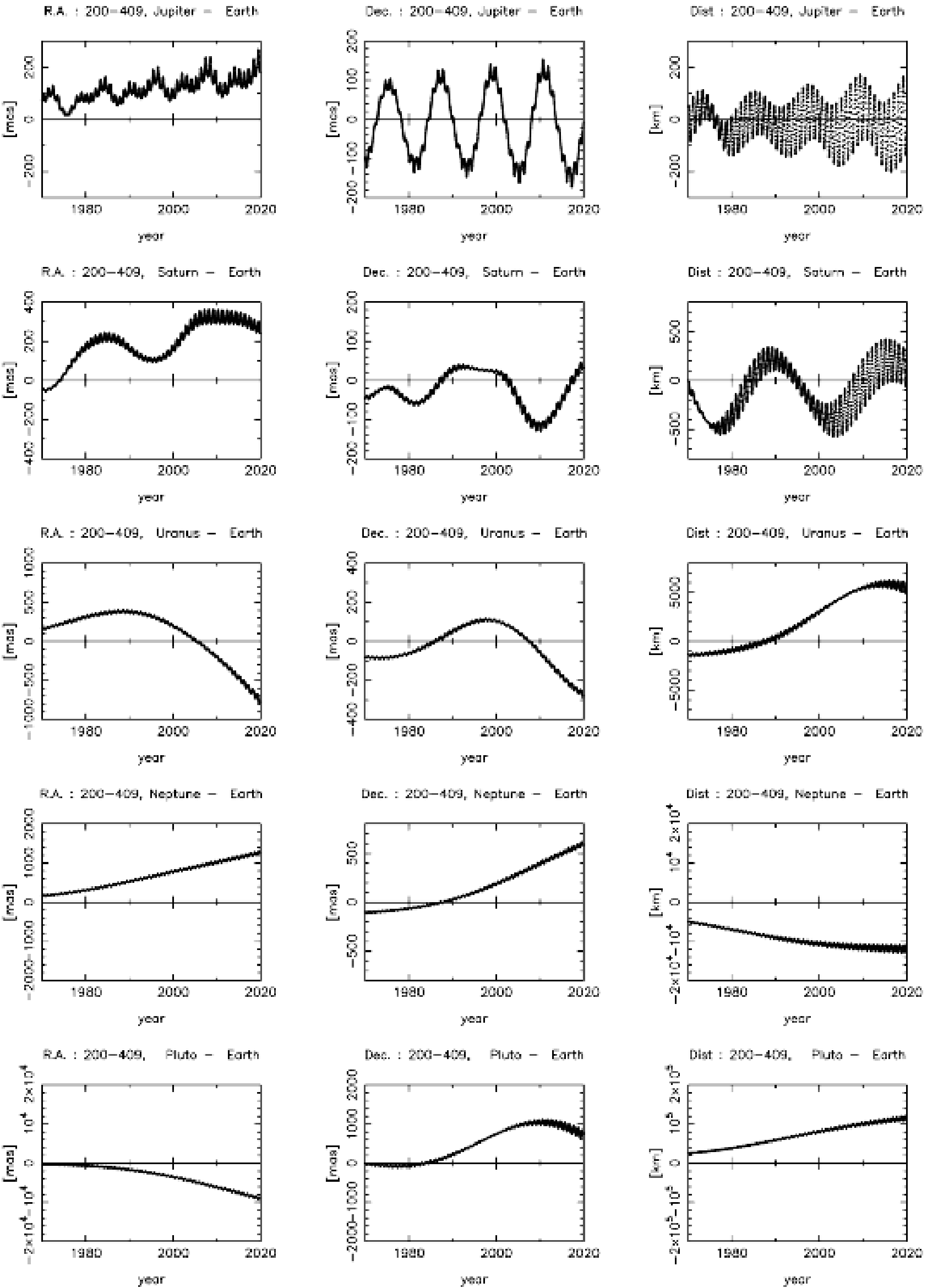

Fig. 2. The outer planet differences, DE200-DE409: effectively the errors in the Astronomical Almanac for the years, $1984-2002$. 
R.A: $405-409$, Nercury $=$ Earth

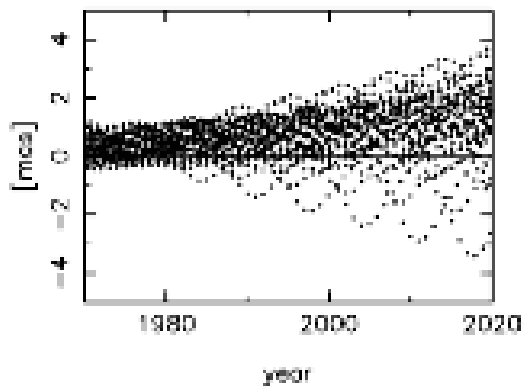

R.A. : 405-409, Venus - Eorth

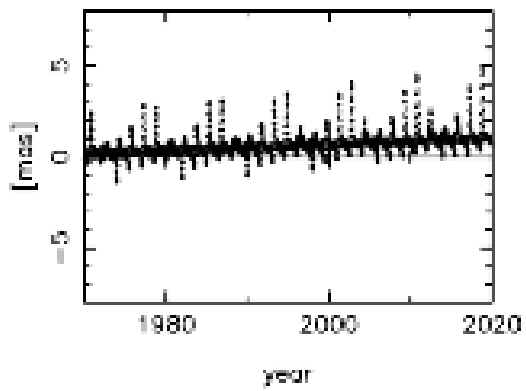

RA : 105-409, The Sun - Earth

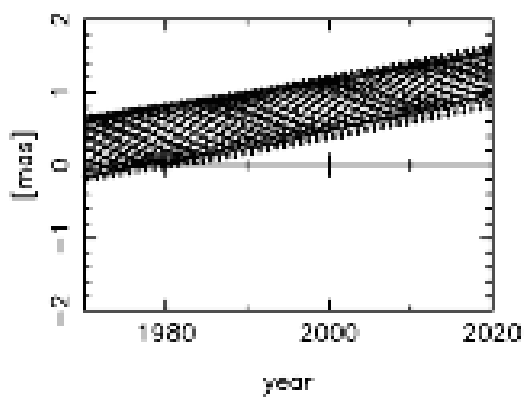

R.A. : 405-409, Wars - Earth

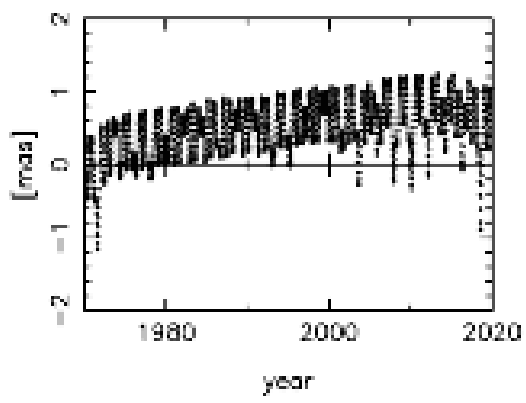

Dec. : 405-409, Nercury = Earth

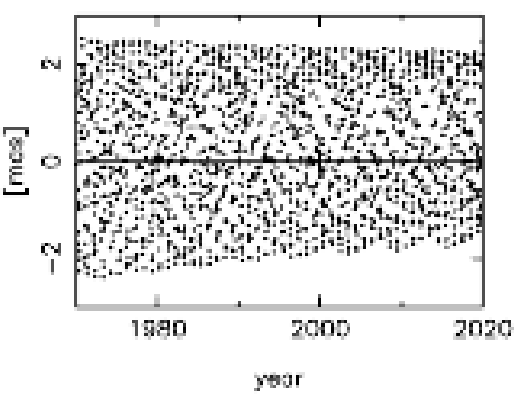

Cec. : 405-409, Venus - Eerth

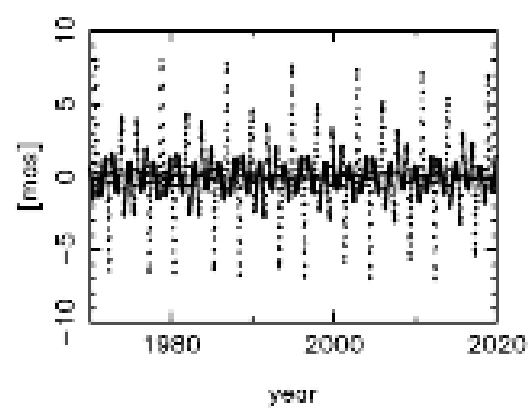

Dec. : 105-109, The Sun - Earth

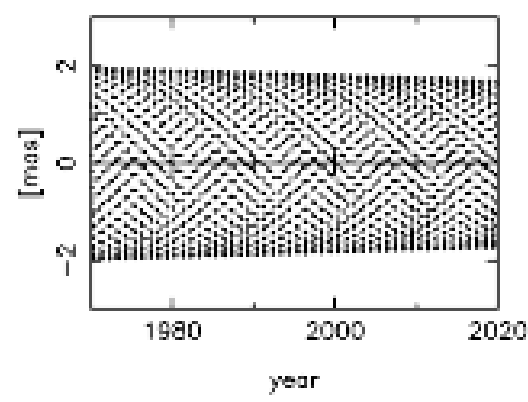

Dec. : 405-409, Mars - Earth

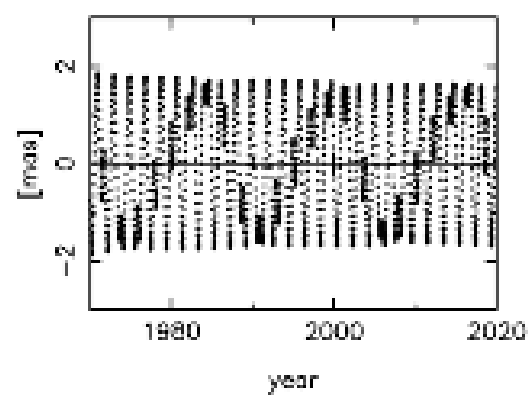

Dist : 405-409, Mercery - Earth

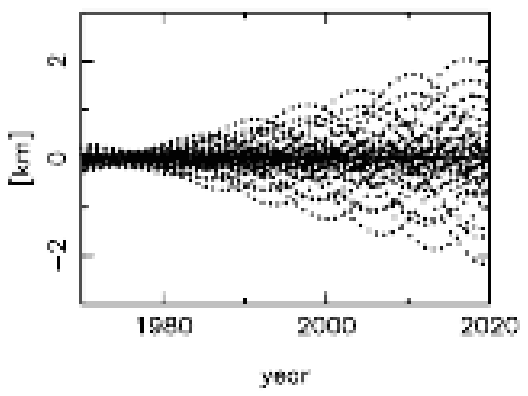

Dist: 405-409, Verus - Ecth

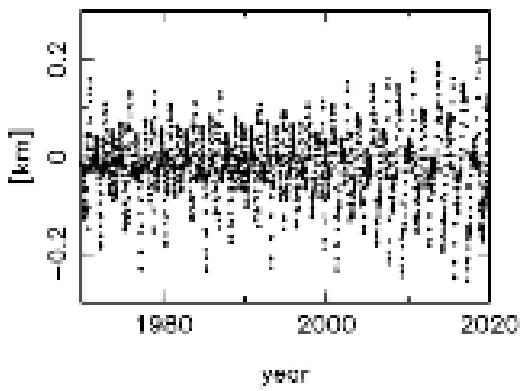

Dist : 405-409. The Sun - Earth

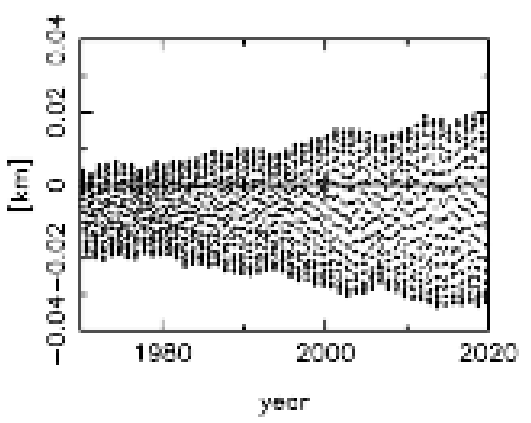

Dist : 405-409, Mars - Earth

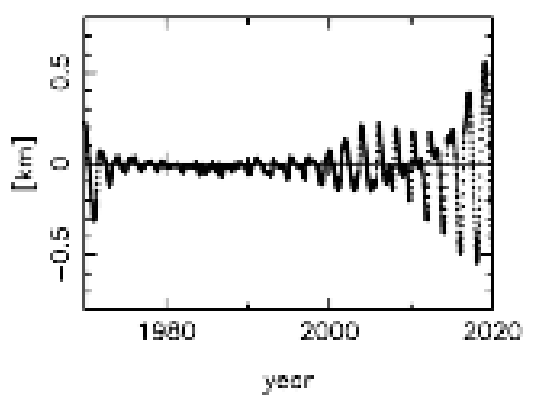

Fig. 3. The inner planet differences, DE405-DE409: estimates of the errors in the Astronomical Almanac starting in the year 2003.

DE405 shows an order of magnitude improvement over DE200, especially for the inner planets. The relative positions and angles are all on the $1 \mathrm{~km}$ level, with the mean motion error being no more that $2 \mathrm{~km} \mathrm{cty}^{-1}$. Some of the scatter in $\Delta \alpha$

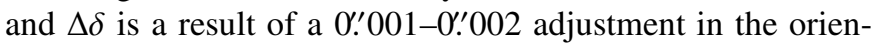
tation, brought by the use of the ICRF-based VLBI at Venus. The subsequent orientations of the Mars and Mercury orbits rely upon the Venus orbit and the tying together of all the inner planet system from the ranging observations. Away from the data time-span, the extrapolation of the DE405 Earth-Mars range has drifted by less than $200 \mathrm{~m}$ at the present time - surprisingly good, considering the expected effects of the asteroid perturbations. For the outer planets, the modern CCD data establish $\alpha$ and $\delta$ directly over the present decade or so; the radial direction is different, however, being highly dependent upon the mean motion (Kepler's third law) which is determined from the whole span of observations. 

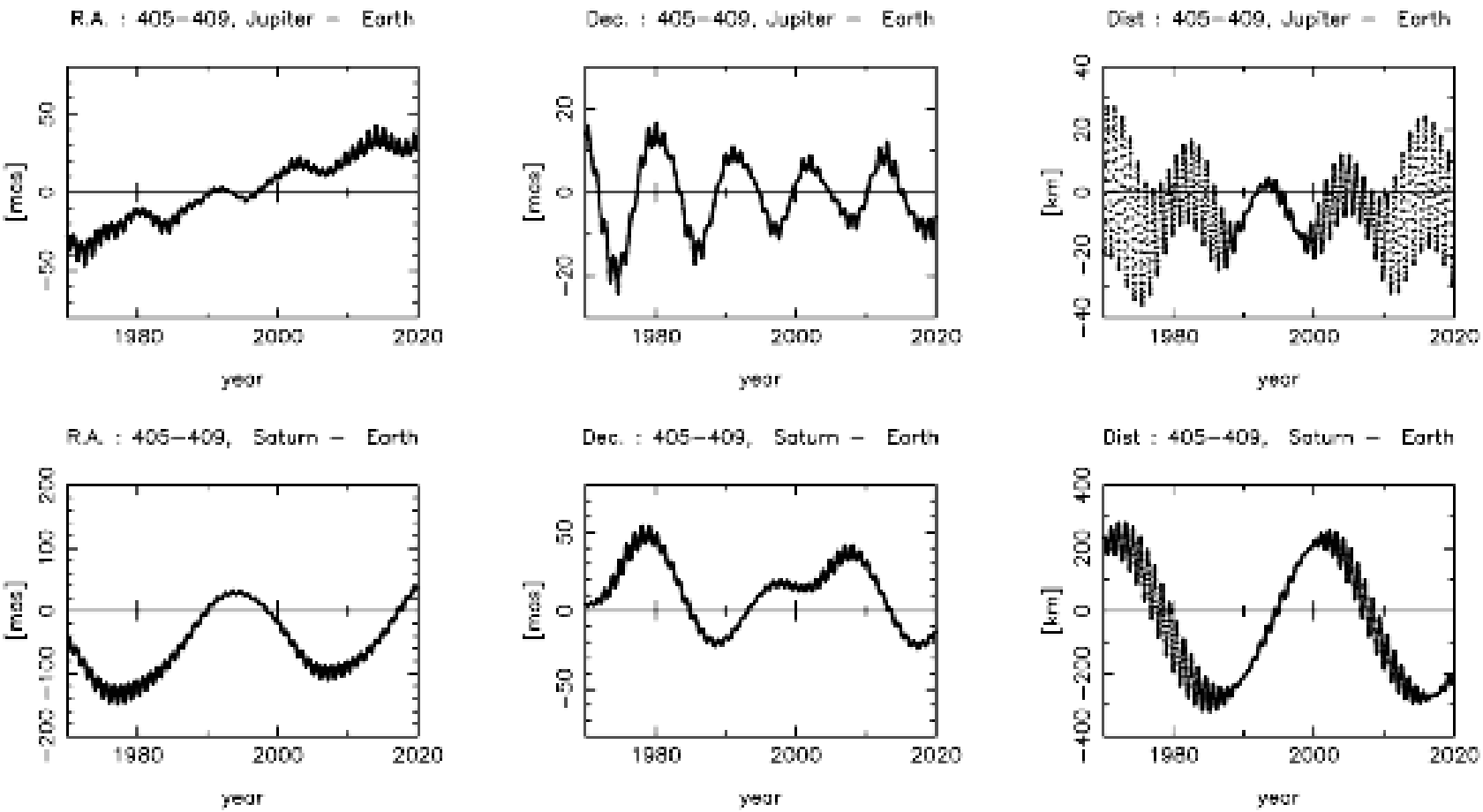

RA : 405-409, Uranus - Ecrth

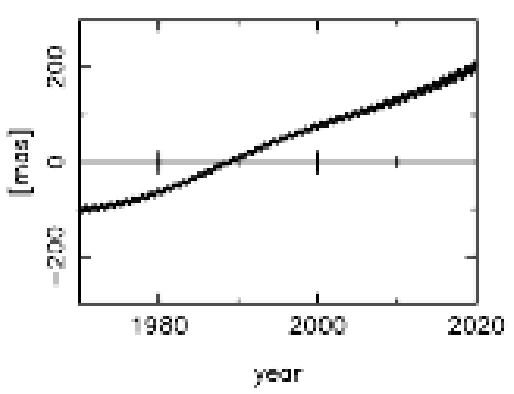

Dec. : $405-409$, Uranus - Eorth
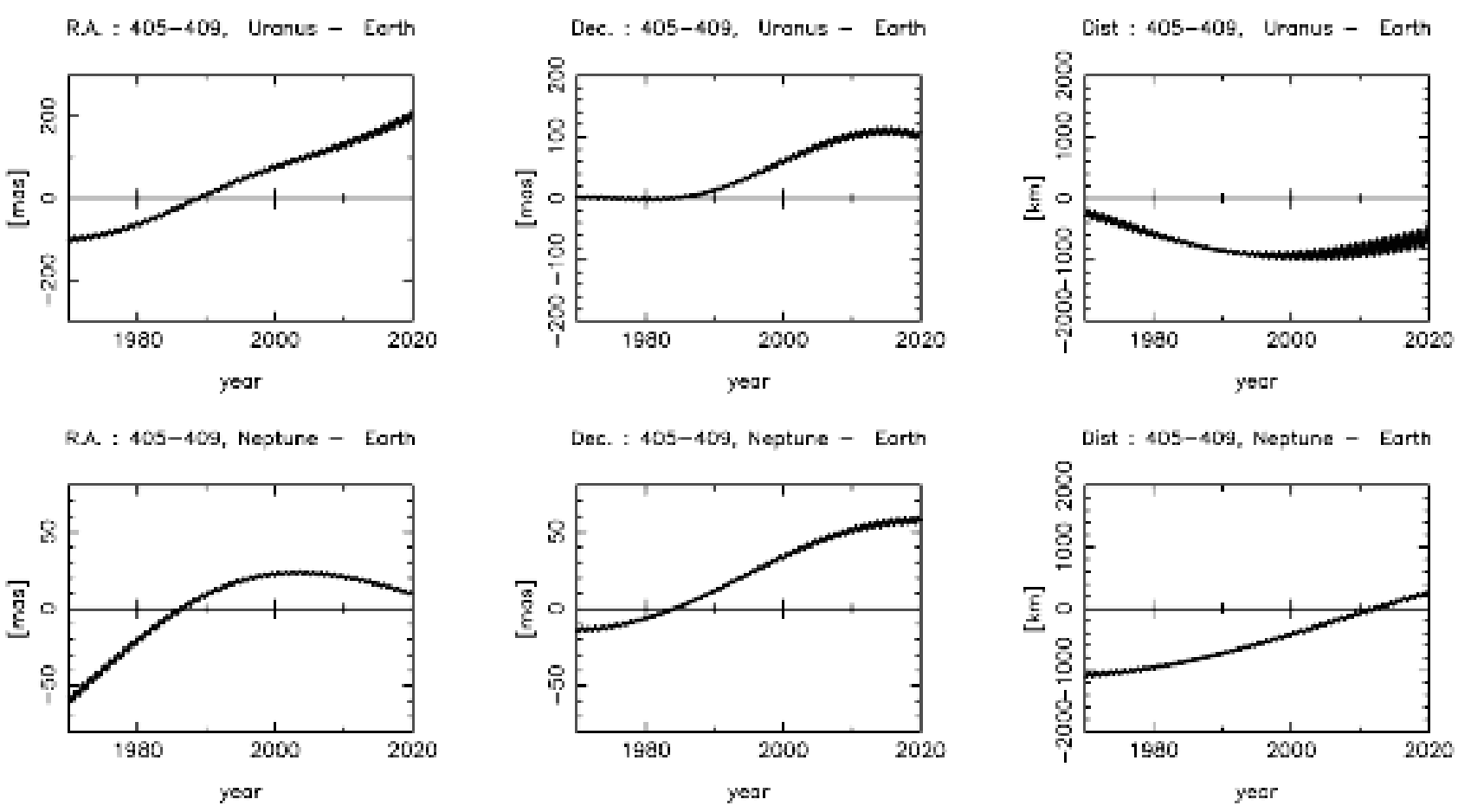

Dec. : 405-409, Nepture - Earth

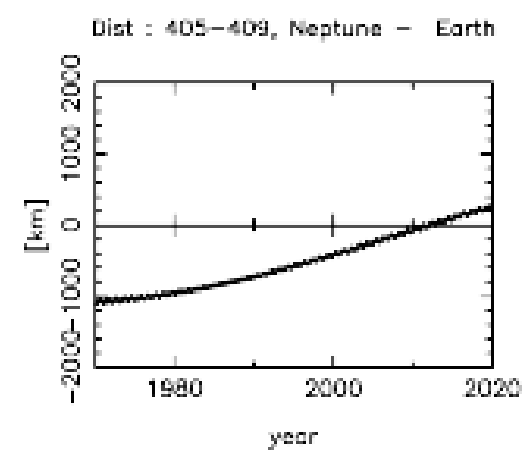

R.4. : 405-409, Fluto - Earth

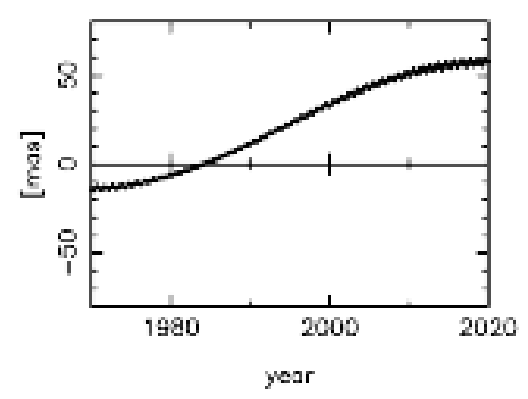

Dec. : 405-409, Pluto - Earth
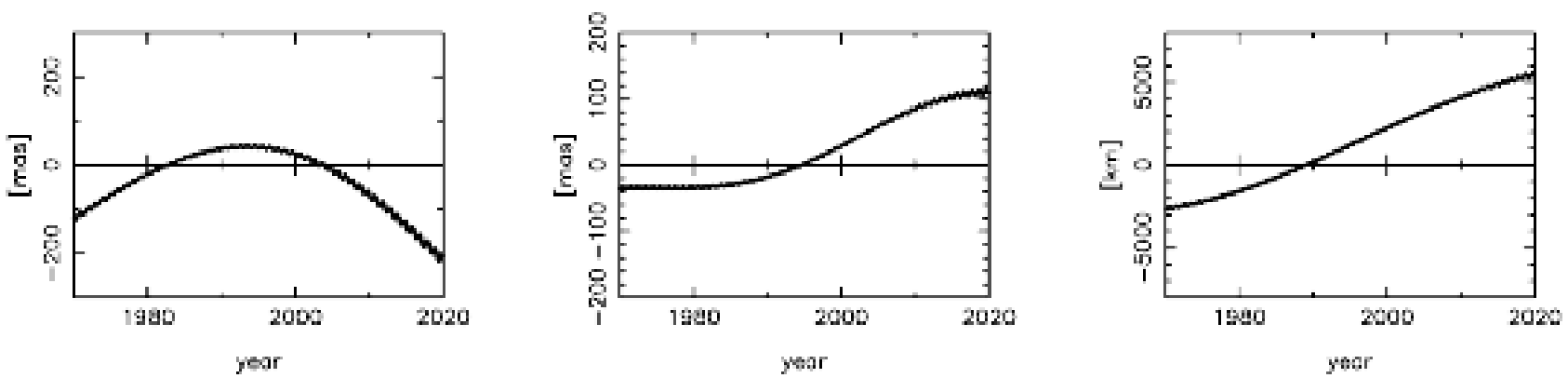

Fig. 4. The outer planet differences, DE405-DE409: estimates of the errors in the Astronomical Almanac starting in the year 2003. 
In summary, the inner planets in DE200 are accurate to about 10-20 km; those in DE405, to 1-2 km. The outer planets show errors which increase outward from the sun: tenths of an arcsecond outward to arcseconds. Only with the recent CCD data are the directions established at the 0 .' 1 level and below.

Acknowledgements. The work described in this paper was carried out at the Jet Propulsion Laboratory, California Institute of Technology, under contract with the National Aeronautics and Space Administration.

\section{References}

Border, J. S. 2003, priv. comm. Folkner, W. M. 1997, priv. comm.
Konopliv, A. S. 2003, priv. comm.

Owen, W. M. 2003, priv. comm.

Standish, E. M. 1982, A\&A, 114, 297

Standish, E. M. 1990, A\&A, 233, 252

Standish, E. M., \& Williams, J. G. 1990, in Inertial Coordinate System on the Sky, ed. J. H. Lieske, \& V. K. Abalakin (Dordrecht: Kluwer Academic Publishers), 173

Standish, E. M. 1998, JPL IOM 312.F-98-048

Standish, E. M., \& Fienga, A. G. 2002, A\&A, 384, 322

Stone, R. C., \& Harris, F. H. 2000, AJ, 119, 1985

Stone, R. C. 2003, priv. comm.

Williams, J. G. 1984, AJ, 57, 1

Williams, J. G., \& Standish, E. M. 1989, in Reference Frames, ed. J. Kovalevsky, I. I. Mueller, \& B. Kolaczek (Dordrecht: Kluwer Academic Publishers) 